thek

\title{
edoc
}

Institutional Repository of the University of Basel

University Library

Schoenbeinstrasse 18-20

CH-4056 Basel, Switzerland

http://edoc.unibas.ch/

Year: 2013

\section{Analysis of neural stem cell self-renewal and differentiation by transgenic RNAi in Drosophila}

\author{
Jiang, Yanrui and Reichert, Heinrich
}

Posted at edoc, University of Basel

Official URL: http://edoc.unibas.ch/dok/A6043865

Originally published as:

Jiang, Yanrui and Reichert, Heinrich. (2013) Analysis of neural stem cell self-renewal and differentiation by transgenic RNAi in Drosophila. Archives of biochemistry and biophysics, Vol. 534, H. 1-2. S. 38-43. 
Analysis of neural stem cell self-renewal and differentiation by transgenic RNAi in Drosophila

Yanrui Jiang*, Heinrich Reichert

Biozentrum, University of Basel, Klingelbergstrasse 50/70, CH-4056 Basel, Switzerland

*Corresponding author

Address: Biozentrum, University of Basel, Klingelbergstrasse 50/70, CH-4056 Basel, Switzerland

Tel: +41612671617 Fax: +41612671613

E-mail addresses: yanrui.jiang@unibas.ch (Y. Jiang), heinrich.reichert@unibas.ch (H. Reichert). 


\begin{abstract}
The fruit fly, Drosophila melanogaster, has proved to be a useful model organism for studying the biology of neural stem cells. Notably, significant progress has been made in identifying the molecular mechanisms that regulate the asymmetric cell divisions of the neural stem cell-like neuroblasts during brain development. Recently, the emerging technology of genome-wide transgenic RNA interference (RNAi), which makes it possible to analyze complicated developmental processes in a targeted, tissue-specific way, has been used for the analysis of gene function in Drosophila neuroblasts. Here, we review the key molecular mechanisms that regulate the asymmetric cell divisions of neuroblasts during brain development in Drosophila. We then summarize recent genome-wide transgenic RNAi screens in Drosophila and report on the identification of new regulators and gene networks that are required in balancing neuroblast selfrenewal and differentiation.
\end{abstract}

Keywords: neural stem cell, neuroblast, self-renewal, differentiation, RNAi, Drosophila 


\section{Highlights}

Neuroblasts are neural stem cells in the Drosophila brain.

Cell fate determinants control self-renewal and differentiation of neuroblasts.

Genome-wide transgenic RNAi identify new genes regulating neuroblasts proliferation.

Drosophila neuroblasts are a new model to study cancer stem cells.

Abbreviations used: aPKC, atypical PKC; Brat, Brain tumor; GMC, ganglion mother cell; INP, intermediate neural progenitor; Insc, Inscuteable; lgl, lethal (2) giant larvae; Loco, Locomotion defects; Mud, Mushroom body defect; Pins, Partner of inscuteable; Pon, Partner of Numb; Pros, Prospero; RISC, RNA-induced silencing complex; RNAi, RNA interference; TRiP, Transgenic RNAi Project; VDRC, Vienna Drosophila RNAi Center. 


\section{Introduction}

The central nervous system in both vertebrates and invertebrates comprises complex networks of neuronal circuits. The neural cells that make up these networks are derived from neural stem cells, which have the ability to self-renew and to give rise to differentiated neuronal and glial progeny through asymmetric and proliferative cell divisions. Balancing self-renewal and differentiation of neural stem cells is essential during development, because dysregulation of genes controlling asymmetric cell division can cause severe developmental defects, and furthermore, lead to the formation of brain tumors [1-3].

In the past two decades, the fruit fly, Drosophila melanogaster, has proved to be a powerful model organism for studying the biology of neural stem cells [4-7]. Drosophila neural stem cells are called neuroblasts for historical reasons. Many important genetic elements and signaling pathways that regulate the asymmetric cell divisions of Drosophila neuroblasts have been characterized and analyzed in great detail $[5,8,9]$. However, our understanding of the molecular and cellular mechanisms controlling self-renewal and differentiation of Drosophila neuroblasts is still incomplete.

Recently, significant progress in analyzing these mechanisms has been attained through the use of genome-wide RNA interference (RNAi) for the identification and functional analysis of genes involved in neuroblast proliferation in the developing brain. This has been possible due to the generation and availability of several independent genome-wide transgenic RNAi libraries, which are based on the binary Ga14/UAS expression system that allow analysis of complicated developmental processes in a targeted, tissue-specific way [10-13].

In this review, we focus on the biology of Drosophila neuroblasts and summarize the key molecular mechanisms that regulate the asymmetric cell divisions of these neuroblasts during 
brain development. We then introduce recent genome-wide RNAi screens in Drosophila. Finally, we review the application of genome-wide transgenic RNAi in the analysis of selfrenewal and differentiation of Drosophila neuroblasts and report on the identification of new regulators and gene networks that are required in balancing neuroblast self-renewal and differentiation.

\section{Neural stem cells in Drosophila}

Neuroblasts, the neural stem cells in Drosophila, have the characteristic feature of the neural stem cells in vertebrates, namely to divide asymmetrically to self-renew while generating a wide range of more differentiated progeny [3-7]. In Drosophila, neuroblasts give rise to differentiated neurons and glia cells in the central brain, optic lobes, and ventral nerve cord [14]. In this review, we will focus primarily on the mechanisms that underlie the specification and homeostasis of the neuroblasts in the central brain; similar mechanisms have been shown to operate in the neuroblasts of the optic lobes and ventral nerve cord, which have been recently reviewed elsewhere $[3,5,6]$.

In Drosophila, neurogenesis starts during early embryonic development [15]. In the embryonic neuroectoderm, groups of cells acquire the potential to become neuroblasts by expressing proneural genes, such as the genes of the achaete-scute complex and daughterless (Fig. 1A) $[15,16]$. From each of these equivalence groups a single cell is selected to become a neuroblast through a process called "lateral inhibition", which is mediated by the Notch signaling (Fig. 1A) [16]. The specified neuroblasts enlarge in size, delaminate basally from the neuroectoderm and then go on to divide asymmetrically along their apical-basal axis in a stem cell-like manner (Fig. 1A) $[6,7]$. 
In the developing brain, neuroblasts undergo two phases of proliferation. The first takes place during embryogenesis, and the neuroblasts only go through a limited number of cell divisions to generate the primary neurons of the larval brain $[14,17,18]$. After a quiescent phase at the end of embryogenesis, most of the brain neuroblasts reinitiate proliferation in a second phase of neurogenesis during larval development to produce numerous secondary neurons in the adult brain [18-21]. These secondary neurons make synaptic interconnections and form adult-specific neuropile structures during pupal development [18,22]. The clonal unit generated by a neuroblast, consisting of primary neurons as well as secondary neurons, is referred to as the neuroblast's lineage [18,22,23].

Approximately 100 neuroblasts have been identified in each hemisphere of the Drosophila brain, and these can be divided into two types (Fig. 1B) [7,8,24-27]. The type I neuroblasts, which constitute the majority of the brain neuroblasts, divide in a simple way. During each division, type I neuroblasts give rise to a larger daughter cell, the renewed neuroblast, and a smaller daughter cell called a ganglion mother cell (GMC). Each GMC divides only once to generate two postmitotic cells that further differentiate into neurons or glia cells (Fig. 2A) [7,8,24]. Recently, a second type of neuroblast, termed a type II neuroblast, has been identified in the Drosophila brain [25-27]. Unlike type I neuroblasts, there are only eight type II neuroblasts in each brain hemisphere. Six type II neuroblasts localize at the dorsomedial edge of the posterior brain lobe, and the other two are formed at a more lateral position (Fig. 1B) [25-27]. In contrast to type I neuroblasts, the type II neuroblasts divide in a more complex way to self-renew and to give rise to an intermediate neural progenitor (INP) cell, which undergoes several rounds of cell division, each of which results in self-renewal of the INP and in the generation of a GMC that further produces two progeny (Fig. 2B) [25-27]. Because of the amplification of proliferation through the divisions of INP cells, a significant increase in neural cell number occurs in type II 
neuroblast lineages. Thus, whereas most type I neuroblast lineages consist of approximately 100 to 150 neurons, type II neuroblasts typically give rise to lineages that are 3 to 5 times larger [25]. In addition, to counter-balance the amplification of proliferation, excess neurons in type II lineages are eliminated through programmed cell death, a process that is essential for the correct neuropile innervation in the adult brain [28].

\section{Asymmetric cell divisions of Drosophila neuroblasts}

Stem cell divisions in Drosophila, as in other animals, can be controlled by cell-intrinsic as well as cell-extrinsic mechanisms, to generate two daughter cells with different cell fates [29]. While the extrinsic regulation of stem cell division has been well-studied in the fly ovaries [30], the asymmetric cell divisions of the neuroblasts appear to be controlled primarily by cell-intrinsic regulatory mechanisms $[8,31]$. This intrinsic regulation of asymmetric neuroblast divisions is achieved by the asymmetric localization of a number of cell fate determinants within the neuroblast during mitosis and subsequently by the segregation of these proteins into only one of the two daughter cells [8,31]. During mitosis, two protein complexes are formed on opposite sides of the neuroblast. On the apical side, the proteins Bazooka, Par-6, atypical PKC (aPKC), Inscuteable (Insc), Partner of inscuteable (Pins), Gai, Locomotion defects (Loco), and Mushroom body defect (Mud) form an apical complex; whereas on the opposite basal side, the cell fate determinants Brain tumor (Brat), Prospero (Pros), and Numb, as well as two adaptor proteins Miranda and Partner of Numb (Pon) are localized in a basal complex [8,31].

In the apical complex, Bazooka, Par-6 and aPKC constitute the Par complex, which already localizes to the apical cortex during interphase and establishes the apical-basal polarity in the neuroblast [32-36]. The Pins, Gai, Loco, and Mud proteins are linked to the Par complex through 
Insc and are required for spindle orientation [37-45]. Although all three proteins of the Par complex are preferentially segregated into the new neuroblast after cell division, and mutations in any of them affect the localization of the other apical proteins and the spindle orientation, the Par complex does not seem to control cell fate directly [8]. Instead, these proteins are thought to exert their function by inducing and restricting cell fate determinant proteins to localize to the basal side of the dividing neuroblast [8].

In Drosophila, three cell fate determinants, Brat, Pros, and Numb, have been characterized and studied in some detail [5-8]. All three proteins asymmetrically localize to the basal cortex during neuroblast division and subsequently are inherited only by the smaller GMC. In the GMC, these proteins are thought to play two essential roles: first, to inhibit cell proliferation by suppressing the expression of neuroblast-specific genes and promoting cell cycle exit; second, to trigger cell differentiation by activating a neural cell differentiative program [5-8]. Numb is a membraneassociated protein and it suppresses the Notch signaling pathway in the GMC $[46,47]$. Pros is a homeodomain containing transcription factor that is asymmetrically segregated into the GMC where it enters the nucleus and activates or represses more than 700 target genes that may be required for neuronal differentiation [48-51]. Brat is a RNA-binding protein that negatively regulates cell growth and ribosomal RNA synthesis [52-55]. Mutations in any of the three determinant genes result in the overproliferation of the neuroblasts and the formation of brain tumors [53-57]. The observation of overgrowth and tumorigenesis in the mutant brains have recently made Drosophila neuroblasts an emerging model to study cancer stem cells (see below) $[8,9,31]$.

Although numerous studies have been made using classic genetic and molecular methods to investigate the functions of the three cell fate determinants in Drosophila neuroblasts, the complete gene networks controlled by these proteins in the regulation of neuroblast self-renewal 
and differentiation are still poorly understood [5-8]. To obtain insight into these gene networks, large-scale genome-wide transgenic RNAi technology has recently been used in order to identify novel genes that are potential regulators in the self-renewal and proliferation of Drosophila neuroblasts $[12,13]$.

\section{Genome-wide RNAi screens in Drosophila}

RNA interference (RNAi) is a cellular process that can sufficiently suppress (knock down) the expression of genes at the post-transcription level. RNAi is mediated by the RNA-induced silencing complex (RISC) and short double-stranded RNA molecules that recognize their target genes in a sequence-specific manner [58,59]. These RNA molecules are either encoded endogenously (microRNAs, siRNAs) or introduced exogenously [60,61]. With the availability of a large number of genome sequences for different organisms, it is possible to construct RNAi libraries that target and silence every individual gene, thus facilitating large-scale genome-wide analysis of gene functions [10]. In the past decade, a variety of RNAi libraries have been generated and screens have been made using different cell cultures or model organisms including C. elegans, Planaria, and Drosophila.

Early RNAi-based screens in Drosophila were mostly carried out in cultured cells, and a wide range of diverse biological processes have been studied in cell culture models [10]. To date, cellbased RNAi screens have been used to identify new candidate genes in genetic networks such as those that regulate growth and viability [62], cell cycle [63], cell death [64], cell signaling [65], and host-pathogen interactions [66]. Although this type of screen has provided new findings for many basic cellular processes, it is not suitable for the analysis of more complex multicellular developmental processes that occur within the intact organism. 
In order to analyze complicated cellular and developmental processes in a tissue-specific manner in the intact animal, a second type of genome-wide screen has been developed which uses transgenic flies carrying RNAi transgenes that can be combined with the established Ga14/UAS binary expression system (Fig. 3) [67]. Currently, three independent sources of genome-wide UAS-RNAi transgenic lines are available [Vienna Drosophila RNAi Center (VDRC), Transgenic RNAi Project (TRiP) at the Harvard Medical School, and NGI-FLY RNAi Resources in Japan], such that altogether about $90 \%$ of the annotated genes in the Drosophila genome can now be targeted by these lines [67]. With the availability of these lines, genome-wide transgenic RNAi screens have been successfully used to study Notch signaling [68,69], muscle morphogenesis and functions [70], host-pathogen interactions [71], and self-renewal of neuroblasts [12,13]. Taken together, these studies show that even very complicated biological mechanisms can be systematically analyzed at the genome-wide level by transgenic RNAi.

\section{Transgenic RNAi analysis of neuroblast self-renewal and proliferation in Drosophila}

The suitability of large-scale genome-wide transgenic RNAi screens for the identification of candidate genes involved in the regulation of self-renewal and proliferation of Drosophila neuroblasts has been demonstrated in two recent reports [12,13]. In the first report, Knoblich and colleagues carried out a genome-wide RNAi screen to indentify genes that are involved in cytokinesis, cell growth, and differentiation in brain neuroblasts and their lineages (Fig. 4) [12]. Knock-down of candidate genes was targeted to the neuroblasts by crossing each individual UAS$R N A i$ line to the driver line insc-Gal4 that is expressed in both type I and type II neuroblasts (Fig. 4A) [54]. In total, 17,362 RNAi lines were screened (corresponding to $89 \%$ of the Drosophila genome). $24.1 \%$ of the lines caused lethality and were further analyzed for brain phenotypes 
(Fig. 4B). This screen identified about 600 candidate genes (832 lines) that cause an abnormal brain phenotype. The genes were then divided into subgroups according to a set of phenotypic categories based on the similarities of the abnormalities in the number, size and shape of the neuroblasts or their daughter cells (GMC, INP, or the entire lineage). For example, among these 600 candidates, a subgroup of 29 genes caused an overproliferation phenotype, suggesting that they are required for restricting neuroblast self-renewal. In addition, based on the annotated gene structure and the known or predicted molecular functions of the candidates, the authors also constructed gene networks using clustering algorithms. In this manner, they identified all putative transcription factors and chromatin regulators that form a transcriptional network for neuroblast self-renewal and also defined genes that interact with known regulators of asymmetric cell divisions and thus form an asymmetric cell division regulatory network.

In a second study, Doe and colleagues combined both transcriptional profiling analysis and transgenic RNAi knock-down experiments, again with the aim of characterizing genes that regulate neuroblast self-renewal in Drosophila [13]. Using microarray-based transcriptome analysis, the authors first studied six different Drosophila mutants, including brat and lethal (2) giant larvae $(\lg l)$, which are known to generate ectopic neuroblasts in the third instar larval brains. By comparing the transcriptional profiling of these six mutants with wild-type, they identified around 1000 genes with elevated expression in the mutant brains, suggesting that they are likely to be expressed in neuroblasts and might be involved in neuroblast homeostasis. To investigate the function of these genes, transgenic RNAi knock-down experiments were carried out in which $U A S-R N A i$ lines were crossed with another neuroblast driver line, wor-Gal4 [72]. This functional analysis yielded a list of 84 potential regulators for neuroblast self-renewal in Drosophila. 
The majority of genes identified in both of these screens are thought to promote neuroblast selfrenewal, as targeted knocking down of these genes generally showed an underproliferation phenotype (538 of 620 genes in [12], and 72 of 84 genes in [13]). Furthermore, for the genes with an underproliferation phenotype, 46 genes were identified by both groups. Interestingly, both screens identified a rather small number of genes for which RNAi knock-down resulted in an increase in the number of neuroblasts (18 in [12], and 12 in [13]). However, among these, only two genes (miranda and Ssrp) were found by both groups. The reason for this low degree of overlap between the two studies is not clear; differential expression patterns of the Gal4 driver lines, different genetic backgrounds of the fly strains used, as well as differences in the design of the two screens might be responsible, at least in part. The altered expression of specific genes was also different among the mutants with ectopic neuroblast phenotypes (with elevated expression of a given gene in one mutant, but decreased expression of the same gene in another), underscoring the fact that proliferation control in neuroblasts is a highly complex process.

In summary, these two recent transgenic RNAi screens have identified a large number of novel genes that appear to play important roles in controlling the balance between self-renewal and differentiation of Drosophila neuroblasts. Although the precise function of each of these genes needs to be validated using classical genetic methods, these studies indeed provide a valuable source of candidate genes for further analysis. Moreover, since a large subset of the candidate genes identified in these screens have conserved orthologs in mammals, further analysis of these genes in murine models is likely to provide new insight into mammalian neural stem cell research as well.

\section{Drosophila neuroblasts as a model to study brain tumors}


The investigation of genes involved in the balance between neuroblast self-renewal and differentiation is important for understanding the mechanisms of neural stem cell action in normal brain development. Moreover, there is an additional motivation for this type of research. In recent years, increasing evidence indicates that a small fraction of stem cells, or stem cell-like progenitor cells, might be the origin of certain cancers (cancer stem cells) including cancers of the brain [73]. However, the molecular mechanisms underlying normal and tumorigenic development in these stem cells are largely unknown. Therefore, understanding how the balance between self-renewal and proliferation is disrupted in tumor stem cells at the cellular and molecular level is likely to be essential for future therapeutic implications both in tumor biology and in stem cell-based regenerative medicine $[8,9]$.

A first Drosophila model for cancer study was established more than three decades ago, when it was shown that mutations in genes such as lethal (1) disc large, lgl, lethal (2) giant disc, and lethal (3) giant larvae can lead to malignant overproliferation in Drosophila brains or discs [74]. More recently, comparable overgrowth phenotypes were also observed in brat, pros, and numb mutant brains, indicating that these cell fate determinants also act as tumor suppressor genes in Drosophila neuroblasts [53,55-57]. Indeed, the tumor suppressor function of genes involved in asymmetric cell division has been unequivocally demonstrated using a transplantation assay in which brain tissue from mutants of brat, pros, or numb transplanted into wild-type adult flies formed large malignant and metastatic tumors that eventually killed the hosts [75].

In view of these findings, it seems apparent that many other genes involved in neuroblast selfrenewal and differentiation may also act as tumor suppressors or oncogenes $[9,75]$. Thus an analysis of the potential role of the candidate genes involved in tumorigenesis from the recent and ongoing transgenic RNAi screens is likely to increase our mechanistic insight into the formation of brain tumors that derive from mutant neuroblasts. Indeed, given that transgenic RNAi can be 
targeted to specific cells via Gal4 drivers and be activated or deactivated at specific times via Ga180 inhibitors, this emerging genetic technology seems to be ideally suited for investigating the molecular mechanisms underlying tumor formation and progression as well as tumor abrogation in the Drosophila neural stem cell model.

\section{Acknowledgements}

This work is supported by the Swiss National Science Foundation (SNSF) and SNSF-NFP63 "Stem cells and regenerative medicine". 


\section{References}

[1] J.J. Breunig, T.F. Haydar, P. Rakic, Neuron 70 (2011) 614-625.

[2] F.T. Merkle, A. Alvarez-Buylla, Curr. Opin. Cell Biol. 18 (2006) 704-709.

[3] A.H. Brand, F.J. Livesay, Neuron 70 (2011) 719-729.

[4] R. Urbach, G.M. Technau, Bioessays 26 (2004) 739-751.

[5] H. Reichert, Results Probl. Cell Differ. 53 (2011) 529-546.

[6] B. Egger, J.M. Chell, A.H. Brand, Philos. Trans. R. Soc. B 363 (2008) 39-56.

[7] C.Q. Doe, Development 135 (2008) 1575-1587.

[8] J.A. Knoblich, Cell 132 (2008) 583-597

[9] K.C. Chang, C. Wang, H. Wang, Bioessays 34 (2012) 301-310.

[10] S. Mohr, C. Bakal, N. Perrimon, Annu. Rev. Biochem. 79 (2010) 37-64.

[11] N. Perrimon, J.Q. Ni, L. Perkins, Cold Spring Harb. Perspect Biol. 2 (2010) a003640.

[12] R.A. Neumüller, C. Richter, A. Fischer, M. Novatchkova, K.G. Neumüller, J.A. Knoblich, Cell Stem Cell 8 (2011) 580-593.

[13] T.D. Carney, M.R. Miller, K.J. Robinson, O.A. Bayraktar, J.A. Osterhout, C.Q. Doe, Dev. Biol. 361 (2012) 137-146.

[14] J.A. Campos-Ortega, V. Hartenstein, The embryonic development of Drosophila melanogaster, second ed., Springer, Heidelberg, 1997. 
[15] J.A. Campos-Ortega, Early neurogenesis in Drosophila melanogaster, in: M. Bate, A.M. Arias (Eds), The development of Drosophila melanogaster, Cold Spring Harbor Laboratory Press, New York, 1993, pp. 1091-1129.

[16] C.S. Goodman, C.Q. Doe, Embryonic development of the Drosophila central nervous system, in: M. Bate, A.M. Arias (Eds), The development of Drosophila melanogaster, Cold Spring Harbor Laboratory Press, New York, 1993, pp. 1131-1205.

[17] V. Hartenstein, E. Rudloff, J.A. Campos-Ortega, Rouxs Arch. Dev. Biol. 196 (1987) 473485.

[18] V. Hartenstein, S. Spindler, W. Pereanu, S. Fung, The development of the Drosophila larval brain, in: G. Technau (Ed), Brain development in Drosophila melanogaster. Landes Bioscience, Austin, 2008, pp. 1-31.

[19] J.W. Truman, M. Bate, Dev. Biol. 125 (1988) 145-157.

[20] A. Prokop, G.M. Technau, Development 111 (1991) 79-88.

[21] K. Ito, Y. Hotta, Dev. Biol. 149 (1992) 134-148.

[22] S.R. Spindler, V. Hartenstein, Dev. Genes Evol. 220 (2010) 1-10.

[23] K. Ito, T. Awasaki, Clonal unit architecture of the adult fly brain, in: G. Technau (Ed), Brain development in Drosophila melanogaster. Landes Bioscience, Austin, 2008, pp. 137-158.

[24] M. Weng, C.Y. Lee, Curr. Opin. Neurobiol. 21 (2011) 36-42.

[25] B. Bello, N. Izergina, E. Caussinus, H. Reichert, Neural Dev. (2008) 3:5.

[26] S.K. Bowman, V. Rolland, J. Betschinger, K.A. Kinesey, G. Emery, J.A. Knoblich, Dev. Cell 14 (2008) 535-546. 
[27] J.Q. Boone, C.Q. Doe, Dev. Neurobiol. 68 (2008) 1185-1195.

[28] Y. Jiang, H. Reichert, Neural Dev. (2012) 7:3.

[29] H.R. Horvitz, I. Herskowitz, Cell 68 (1992) 237-255.

[30] L. Li, T. Xie, Annu. Rev. Cell Dev. Biol. 21 (2005) 605-631.

[31] R.A. Neumüller, J.A. Knoblich, Genes Dev 23 (2009) 2675-2699.

[32] A. Wodarz, A. Ramrath, U. Kuchinke, E. Knust, Nature 402 (1999) 544-547.

[33] M. Schober, M. Schaefer, J.A. Knoblich, Nature 402 (1999) 548-551.

[34] M. Petronczki, J.A. Knoblich, Nat Cell Biol. 3 (2001) 43-49.

[35] A. Wodarz, A. Ramrath, A. Grimm, E. Knust, J. Cell Biol. 150 (2000) 1361-1374.

[36] M.M. Rolls, R. Albertson, H.P. Shih, C.Y. Lee, C.Q. Doe, J. Cell Biol. 163 (2003) 10891098.

[37] M, Schaefer, M. Petronczki, D. Dorner, M. Forte, J.A. Knoblich, Cell 107 (2001) 183-194.

[38] F. Yu, Y. Cai, R. Kaushik, X. Yang, W. Chia, J. Cell Biol. 162 (2003) 623-633.

[39] Y. Izumi, N. Ohta, A. Itoh-Furuya, N. Fuse, F. Matsuzaki, J. Cell Biol. 164 (2004) 729-738.

[40] M. Schaefer, A. Shevchenko, A. Shevchenko, J.A. Knoblich, Curr. Biol. 10 (2000) 353-362.

[41] F. Yu, X. Morin, Y. Cai, X. Yang, W. Chia, Cell 100 (2000) 399-409.

[42] F. Yu, H. Wang, H. Qian, R. Kaushik, M. Bownes, X. Yang, W. Chia, Genes Dev. 19 (2005) $1341-1353$.

[43] S.K. Bowman, R.A. Neumuller, M. Novatchkova, Q. Du, J.A. Knoblich, Dev. Cell 10 (2006) 731-742. 
[44] Y. Izumi, N. Ohta, K. Hisata, T. Raabe, F. Matsuzaki, Nat. Cell Biol. 8 (2006) 586-593.

[45] K.H. Siller, C. Cabernard, C.Q. Doe, Nat. Cell Biol. 8 (2006) 594-600.

[46] M.S. Rhyu, L.Y. Jan, Y.N. Jan Cell 76 (1994) 477-491.

[47] E.P. Spana, C. Kopczynski, C.S. Goodman, C.Q. Doe, Development 121 (1995) 3489-3494.

[48] J. Hirata, H. Nakagoshi, Y. Nabeshima, F. Matsuzaki, Nature 377 (1995) 627-630.

[49] J.A. Knoblich, L.Y. Jan, Y.N. Jan, Nature 377 (1995) 624-627.

[50] E.P. Spana, C.Q. Doe, Development 121 (1995) 3187-3195.

[51] S.P. Choksi, T.D. Southall, T. Bossing, K. Edoff, E. de Wit, B.E. Fischer, B. van Steensel, G. Micklem, A.H. Brand, Dev. Cell 11 (2006) 775-789.

[52] D.J. Frank, B.A. Edgar, M.B. Roth, Development 129 (2002) 399-407.

[53] B. Bello, H. Reichert, F. Hirth, Development 133 (2006) 2639-2648.

[54] J. Betschinger, K. Mechtler, J.A. Knoblich, Cell 124 (2006) 1241-1253.

[55] C.Y. Lee, B.D. Wilkinson, S.E. Siegrist, R.P. Wharton, C.Q. Doe, Dev. Cell 10 (2006) 441449.

[56] C.Y. Lee, R.O. Andersen, C. Cabernard, L. Manning, K.D. Tran, M.J. Lanskey, A. Bashirullah, C.Q. Doe, Genes Dev. 20 (2006) 3464-3474.

[57] H. Wang, G.W. Somers, A. Bashirullah, U. Heberlein, F. Yu, W. Chia, Genes Dev. 20 (2006) 3453-3463.

[58] A. Fire, S. Xu, M.K. Montgomery, S.A. Kostas, S.E. Driver, C.C. Mello. Nature 391(1998) 806-811.

[59] P.D. Zamore, T. Tuschl, P.A. Sharp, D.P. Bartel. Cell 101 (2000) 25-33. 
[60] D.P. Bartel, Cell 136 (2009) 215-233.

[61] R.W. Carthew, E.J. Sontheimer, Cell 136 (2009) 642-655.

[62] M. Boutros, A.A. Kiger, S. Armknecht, K. Kerr, M. Hild, B. Koch, S.A. Haas, Heidelberg Fly Array Consortium, R. Paro, N. Perrimon, Science 303 (2004) 832-835.

[63] M. Bjorklund, M. Taipale, M. Varjosalo, J. Saharinen, J. Lahdenpera, J. Taipale, Nature 439 (2006) 1009-1013.

[64] C.H. Yi, D.K. Sogah, M. Boyce, A. Degterev, D.E. Christofferson, J. Yuan, J. Cell Biol. 179 (2007) 619-626.

[65] R. DasGupta, A. Kaykas, R.T. Moon, N. Perrimon, Science 308 (2005) 826-833.

[66] H. Agaisse, L.S. Burrack, J.A. Philips, E.J. Rubin, N. Perrimon, D.E. Higgins, Science 309 (2005) 1248-1251.

[67] G. Dietzl, D. Chen, F. Schnorrer, K.C. Su, Y. Barinova, M. Fellner, B. Gasser, K. Kinsey, S. Oppel, S. Scheiblauer, A. Couto, V. Marra, K. Keleman, B.J. Dickson, Nature 448 (2007) 151156.

[68] J.L. Mummery-Widmer, M. Yamazaki, T. Stoeger, M. Novatchkova, S. Bhalerao, D. Chen, G. Dietzl, B.J. Dickson, J.A. Knoblich, Nature 458 (2009) 987-992.

[69] A. Saj, Z. Arziman, D. Stempfle, W. van Belle, U. Sauder, T. Horn, M. Dürrenberger, R. Paro, M. Boutros, G. Merdes, Dev. Cell 18 (2010) 862-876.

[70] F. Schnorrer, C. Schönbauer, C.C. Langer, G. Dietzl, M. Novatchkova, K. Schernhuber, M. Fellner, A. Azaryan, M. Radolf, A. Stark, K. Keleman, B.J. Dickson, Nature 464 (2010) 287291. 
[71] S.J. Cronin, N.T. Nehme, S. Limmer, S. Liegeois, J.A. Pospisilik, D. Schramek, A. Leibbrandt, M. Simoes Rde, S. Gruber, U. Puc, I. Ebersberger, T. Zoranovic, G.G. Neely, A. von Haeseler, D. Ferrandon, J.M. Penninger, Science 325 (2009) 340-343.

[72] R. Albertson, C. Chabu, A. Sheehan, C.Q. Doe, J. Cell Sci. 117 (2004) 6061-6070.

[73] T. Reya, S.J. Morrison, M.F. Clarke, I.L. Weissman, Nature 414 (2001) 105-111.

[74] E.Gateff, Science 200 (1978) 1448-1459.

[75] E. Caussinus, C. Gonzalez, Nat. Genet. 37 (2005) 1125-1129. 


\section{Figure Legends}

Fig. 1. (A) Specification of the neuroblasts during development. In the neuroectoderm, a group of cells acquire the potential to become a neuroblast by expressing proneural genes (green). Later, a single cell is selected to become a neuroblast through a Notch signaling mediated process called "lateral inhibition" (arrows). The specified neuroblast enlarges in size, delaminates basally from the neuroectoderm and then divides asymmetrically along the apical-basal axis. Schematic representation of the Drosophila larval central nervous system, which consists of the central brain (CB), the optic lobes (OL), and the ventral nerve cord (VNC). The CB contains two types of neuroblasts (NBs). While the majorities are type I NBs (red), eight type II NBs are present in each brain hemisphere (blue).

Fig. 2. (A) Mode of cell division of type I neuroblasts. The neuroblast (NB, pink) divides to self-renew and to give rise to a smaller ganglion mother cell (GMC, orange), which only divides once to generate two postmitotic neurons or glia cells (red). (B) Mode of cell division of type II neuroblasts. The neuroblast $(\mathrm{NB}$, blue) divides to self-renew and to give rise to an intermediate neural progenitor cell (INP, light blue), which undergoes several rounds of cell division, each of which results in self-renewal of the INP and in the generation of a GMC (purple) that further produces two progeny (dark blue).

Fig. 3. Schematic representation of transgenic RNAi in Drosophila. The driver line expresses the yeast protein Gal4, which is under the spatial and temporal control of a specific enhancer. The responder line contains an RNAi construct that is controlled by the UAS. After crossing the 
two lines, Gal4 binds to UAS and leads to the transcription of the RNAi inverted repeat. The RNA hairpins are further processed through the RNAi pathway to generate functional RNAi molecules, which results in the tissue-specific target gene knock-down.

Fig. 4. (A) Simplified workflow of the genome-wide transgenic RNAi screen in Drosophila neuroblasts [12]. The driver line co-expresses Dicer-2 to enhance the transgenic RNAi effect. Schematics show an overproliferative phenotype (left) and a normal brain (right). (B) Summary of the primary screen in [12]. In total, 17,362 lines were screened and $24.1 \%(4,182$ lines) resulted in lethality. In these lethal lines, 832 showed abnormal brain phenotype. 